\title{
RESEARCH
}

Open Access

\section{Added value of contrast-enhanced spectral mammography in symptomatic patients with dense breasts}

\author{
Sara Ahmed Sadek Mohamed*i(B), Sherine George Moftah, Nivine Abd El Moneim Chalabi and \\ Mona Ali Abdel-Wahed Salem
}

\begin{abstract}
Background: Breast cancer is the most common malignancy in females around the world representing $25.1 \%$ of all cancers.

The high prevalence and need for early treatment of breast malignancy highlight the importance of early and accurate diagnosis. In order to achieve this target, it is necessary to select the most appropriate modality for investigation.

Early detection of breast cancer by conventional mammography tends to reduce mortality; however, it has a low sensitivity and specificity in young females with dense breasts owing to reduced contrast between a possible tumor and the surrounding breast tissue with superimposition of the glandular tissue obscuring underlying lesions. Our study included 25 patients with dense breasts presented with different breast symptoms, yet the breast lump was the most common complaint. The aim of our study is to evaluate the supplementary value of contrastenhanced spectral mammography in the assessment of symptomatic patients with dense breasts.

Results: In our study, the enrolled subjects underwent both contrast-enhanced spectral mammography (CESM) and conventional full-field digital mammography (FFDM). CESM was shown to be better than FFDM in terms of sensitivity, positive predictive value, negative predictive value, and accuracy, measuring 100\%,77.8\%, 100\%, and $84 \%$, compared to $56 \%, 75 \%, 46 \%$, and $60 \%$, respectively, yet both modalities showed low specificity, measuring $63.6 \%$ and $66.6 \%$ for CESM and FFDM, respectively.

The added value of CESM was assessed in terms of ability to detect and correctly characterize the lesions in correlation to histopathological results where CESM could detect $88 \%$ of the lesions included in our study and correctly characterized $84 \%$ of the lesions; on the other side, FFDM detected only $20 \%$ of the lesions and correctly characterized $60 \%$ of the lesions. CESM changed the treatment plan to a more extensive surgery $+/$ - neoadjuvant chemotherapy in $57 \%$ out of fourteen cases diagnosed with breast cancer emphasizing the role of CESM in assessing the extent of the disease, multicentricity, and multifocality and consequently tailoring the most appropriate treatment plan suitable for each patient.

Conclusion: Contrast-enhanced spectral mammography is superior to full-field digital mammography in patients with dense breasts with a significant supplementary value in detection, characterization of lesions, and tailoring the appropriate treatment plan.
\end{abstract}

Keywords: Contrast-enhanced spectral mammography, Full-field digital mammography, Dense breast, Breast cancer

*Correspondence: sarasadek890@gmail.com

Radiodiagnosis Department, Faculty of Medicine, Ain Shams University, Cairo,

Egypt

(c) The Author(s). 2021 Open Access This article is licensed under a Creative Commons Attribution 4.0 International License, which permits use, sharing, adaptation, distribution and reproduction in any medium or format, as long as you give appropriate credit to the original author(s) and the source, provide a link to the Creative Commons licence, and indicate if changes were made. The images or other third party material in this article are included in the article's Creative Commons licence, unless indicated otherwise in a credit line to the material. If material is not included in the article's Creative Commons licence and your intended use is not permitted by statutory regulation or exceeds the permitted use, you will need to obtain permission directly from the copyright holder. To view a copy of this licence, visit http://creativecommons.org/licenses/by/4.0/. 


\section{Background}

The incidence of breast cancer is higher in developed countries, while relative mortality is greater in developing countries. Plans for the control of breast cancer should be a priority for health policy makers as breast cancer is the most common malignancy in females worldwide [1].

Conventional mammography is proven to reduce mortality by early detection of breast cancer; however, in patients with dense breasts, its sensitivity and specificity are significantly decreased due to superimposition of the glandular tissue possibly obscuring underlying tumors [2]. Sensitivity of mammography decreases from $87.0 \%$ in women with fatty breasts to $62.9 \%$ in women with dense breasts which allows for a greater number of missed cancers. It is worth mentioning that dense breast has also been reported to be a strong independent risk factor of breast cancer [3].

Treatment options for breast cancer depend on the size of the main tumor as well as the presence or absence of additional foci. Therefore, an accurate estimation of the tumor's size is crucial for selecting the best treatment strategy for each patient. MRI is considered the best imaging investigation for the detection of breast cancer and the assessment of the disease extent in preoperative planning, yet due to the high costs and limited availability, MRI is only performed in a very limited number of cases [2].

The introduction of full-field digital mammography (FFDM) has sparked the development of other techniques that are less expensive than MRI and more widely available such as tomosynthesis and contrast-enhanced spectral mammography (CESM). CESM improves the sensitivity for breast cancer detection without significantly decreasing specificity as it provides higher contrast and better lesion delineation than mammography alone [4].

Contrast-enhanced mammography is a technique based on dual-energy acquisitions, where two images are acquired using conventional low-energy mammography and high-energy mammography following intravenous injection of an iodinated contrast medium. The differences between X-ray attenuation of iodine and breast tissues at these two energy levels are exploited leading to suppression of the background breast tissue to highlight hypervascular lesions. This technique holds the potential for better detection of malignant lesions within dense breast tissue [5].

Preliminary results with CESM examination suggest that similar to breast MRI, CESM is of particular interest for the assessment of the extent of disease, allowing better evaluation of lesion size and detecting more multifocal breast cancers than mammography alone or with ultrasonography combination. Moreover, it is more available and cost-effective than MRI [2].
In our study, we aim to evaluate the supplementary value of contrast-enhanced spectral mammography in the assessment of symptomatic patients with dense breasts.

\section{Methods}

From July 2018 to July 2019, 25 females with ages ranging from 21 to 66 years (mean age 41) were chosen from the breast surgery clinic at El Demerdash Hospital. Pregnant patients, patients with a history of previous allergic reaction to contrast agents, or patients with renal failure were excluded. A full history was obtained. Written informed consent for performing contrast-enhanced spectral mammography (CESM) was obtained. The study was approved by the ethical committee and the institutional review board.

According to the BI-RADS system, seven cases were categorized as BIRADS 3, nine patients as BIRADS 4, one patient as BIRADS 5, and eight patients as BIRADS 6. All patients underwent CESM followed by a targeted ultrasound if enhancing lesions were found. Tissue core biopsies were obtained after CESM except for BIRADS 6 patients who already had histopathologically proven breast cancer and CESM was requested to assess the extent of the disease. Dual-energy CESM acquisitions were done using the Senographe Pristina, GE healthcare contrast-enhanced mammography device.

\section{Patient preparation}

Peripheral IV access was obtained in the antecubital fossa contralateral to the breast of concern with intravenous injection of a non-ionic contrast agent (Ultravist 300 or Omnipaque 300) at a dose of $1.5 \mathrm{ml} / \mathrm{kg}$.

\section{Image acquisition}

A pair of high-energy and low-energy images were acquired in rapid succession and used to make the final image, via a weighted logarithmic subtraction. Both images were acquired after the contrast injection by about 2 min after the end of the injection to allow the contrast to be taken up in the tissue. The standard positions CC and MLO were obtained, with low- and high-energy images acquired in each view, with compression. Image acquisition was done in the following sequence; $\mathrm{CC}$ of the normal breast, CC of the affected breast, MLO of the affected breast, and finally MLO of the normal breast.

The duration of the procedure was approximately 10 min. The final dual-energy subtraction image equalizes the density of fibroglandular tissue and fat, thereby minimizing the visibility of the breast tissue and increasing the conspicuity of the iodinated contrast agent.

The high- and low-energy beams are created by adjusting the peak kilovoltage $(\mathrm{kVp})$ of the $\mathrm{X}$-ray tube and changing the filtration. KVp values between 28 and 
32 are typically used for the low-energy beam, and those between 45 and 49 are typically used for the high-energy beam. In addition, extra filtration, typically copper, is added to the high-energy beam to further harden it.

\section{Image interpretation}

Image analysis and interpretation were done by an experienced breast radiologist. Regarding mammographic examination, the lesions were evaluated according to the Breast Imaging Reporting and Data System (BI-RADS) lexicon designed by the American College of Radiology(ACR), site of the lesions, margin, definition, and \pm calcifications. In the recombined images, MLO and CC views were mainly assessed for the presence or absence of enhancement as well as the type of enhancement (mass or non-mass) and its degree and homogeneity.

Ultrasound-guided tissue core biopsies were obtained in all cases with clinically palpable lumps regardless of their enhancement criteria on CESM as histopathology was considered the gold standard in our study. Concordance between the diagnosis postulated by CESM and the final histopathological results was evaluated. The value of tissue core biopsy was specially highlighted in confirming the malignant nature of BIRADS 4 or 5 lesions and excluding phyllodes tumor in fibroadenomas with atypical features on ultrasound as cystic degeneration (Fig. 1) or large size with no previous studies for comparison. Aspiration for complicated cysts was also done to relieve the patient's pain.

The recombined image should be evaluated in conjunction with the low-energy image. The recombined image is an adjunct to the low-energy image rather than a replacement.

\section{Classification of enhancing lesions}

According to the MRI BI-RADS lexicon, enhancing breast lesions identified on the CESM subtraction images are classified into the following.

\section{Focus}

A focus is a breast lesion $<5 \mathrm{~mm}$. Enhancing focus morphology descriptors include the number (single or multiple), unilateral or bilateral, and intensity of enhancement (faint or intense enhancement). Multiple, bilateral, and faintly enhancing foci are considered as morphology descriptors of benign breast lesions while single, unilateral, or intensely enhancing foci are considered as morphology descriptors of malignant breast lesions [6].

\section{Mass}

A mass is a three-dimensional space-occupying lesion. Morphology descriptors of enhancing mass lesions include shape (round, oval, or irregular), margin
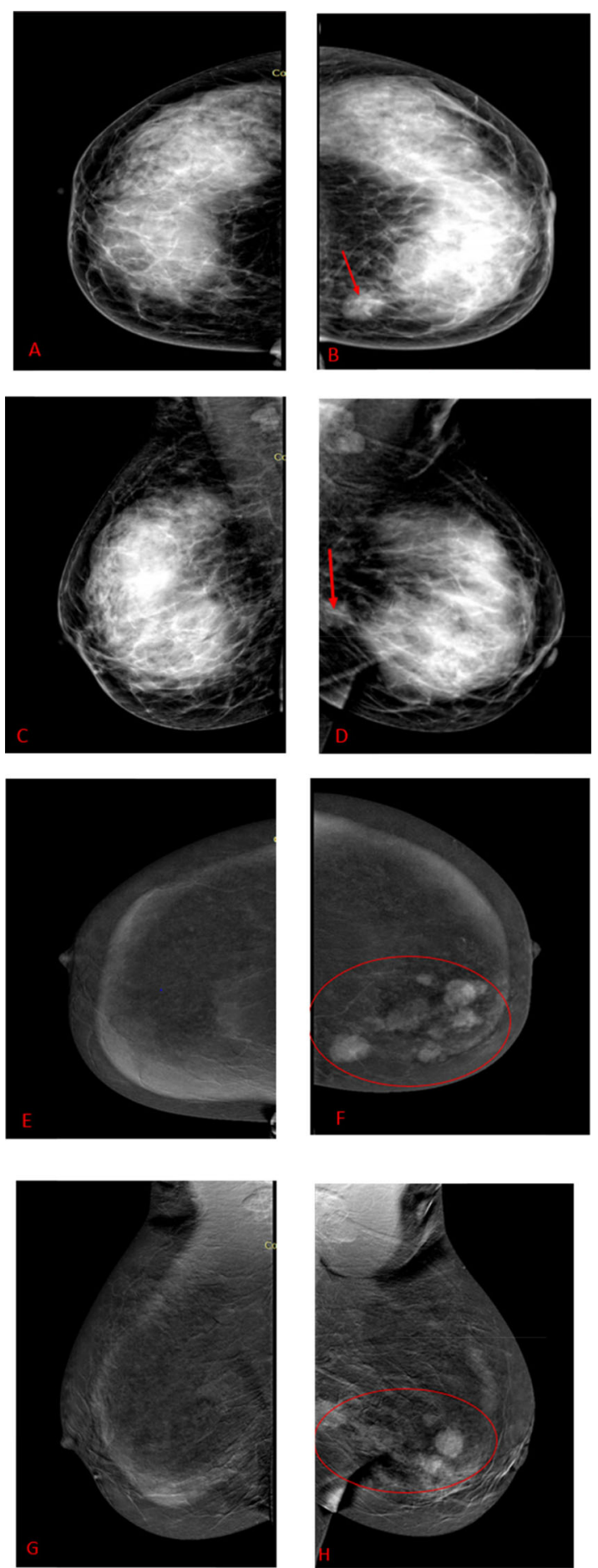

Fig. 1 A 47-year-old female patient presented with right breast lump of 5 months duration. FFDM of both breasts in CC ( $\mathbf{a}$ and $\mathbf{b})$ and MLO (c and d) showing heterogeneously dense breast (ACR C) with right oval shape obscured margin mass seen better in the MLO view (arrowed in c). Recombined images (RI) showing right UOQ intensely enhancing well-circumscribed oval-shaped mass with non-enhancing septations (arrowed in $\mathbf{e}$ and $\mathbf{g}$ ), targeted ultrasound of this lesion revealed hypoechoic well-circumscribed mass with cystic component (i). Biopsy was taken and histopathology revealed fibroadenoma 
circumscribed, and non-circumscribed (irregular or spiculated) and internal enhancement characteristics (homogeneous, heterogeneous, rim enhancement, and dark internal septations). Rounded or oval-shaped mass lesions, circumscribed margins, homogeneous enhancement, and dark internal septations are considered as morphology descriptors of benign lesions while irregular-shaped mass lesions, non-circumscribed margins, heterogeneous, and rim enhancement patterns are considered as morphology descriptors of malignant lesions [6].

\section{Non-mass}

Non-mass enhancement (NME) is non-space occupying lesion. NME morphology descriptors include symmetry (symmetric or asymmetric when comparing both breasts), distribution (focal, linear, segmental, regional, multiple regions, or diffuse), internal enhancement pattern (homogeneous, heterogeneous, clumped, and clustered ring), intensity of enhancement (faint, moderate, or intense). Symmetric NME taking either multiple regions or diffuse distribution and showing homogeneous internal enhancement characteristics are considered as morphology descriptors of benign lesions while asymmetric NME taking focal, linear, segmental, or regional distribution and showing heterogeneous, clumped, or clustered ring internal enhancement characteristics are considered as morphology descriptors of malignant lesions [6].

\section{Statistical analysis}

Data were coded and entered using the statistical package SPSS version 25. It was summarized using descriptive statistics.

Inferential statistical analyses for assessment of the relation between categorical variables were performed using both contingency tables with chi-squared tests for observed and expected values and testing for correlation using the spearman-rho test. All tests were considered statistically significant at a $p$ value equal to or less than 0.05 .

Diagnostic performance indices including sensitivity, specificity, positive, and negative predictive values and overall accuracy were calculated using contingency tables.

Data was presented by graphs, bar charts, and pie charts as well as tables.

\section{Results}

This study included twenty-five female patients with fifty-six breast lesions, with ages between 21 and 66 years. Twenty female patients with dense breasts were classified as (ACR C) and five patients classified as (ACR D). Among these twenty-five patients, fourteen patients had malignant lesions and eleven patients had benign lesions with the final histopathological diagnoses detailed in (Table 1).

As regards the BIRADS given to the detected lesions by FFDM and targeted ultrasound (US), seven patients were categorized as BIRADS $3(28 \%)$, nine patients as BIRADS $4(36 \%)$, one patient as BIRADS 5 (4\%), and eight patients as BIRADS 6 (32\%).

Regarding BIRADS categorization after CESM, the results were different from these obtained using FFDM and US; one patient was categorized as BIRADS $1(4 \%)$, six patients as BIRADS $3(24 \%)$, four patients as BIRADS $4(16 \%)$, six patients as BIRADS $5(24 \%)$, and eight patients as BIRADS 6 (32\%). The BIRADS categorization made after adding CESM to FFDM was strongly correlated with the final BIRADS categorization after histopathology $(P<0.001)$.

The pattern of enhancement of the lesions in the recombined images was classified into mass enhancement in fourteen cases (56\%) and non-mass enhancement in three cases (12\%). Mass and non-mass enhancement, together, were encountered in five cases $(20 \%)$, and in three cases $(12 \%)$, there was no enhancement. Enhancement pattern was not related to the final histopathological diagnosis $(P$ value $>0.05)$.

In order to assess the added value of CESM numerically, the value of CESM was assessed from two aspects; the ability to detect lesions and the ability to characterize lesions as benign or malignant (Tables 2 and 3).

Regarding the ability of CESM in lesion detection including the primary tumor and surrounding satellites, CESM was able to detect all the lesions in twenty-two cases (88\%), eight cases with benign lesions, and fourteen cases with malignant lesions. Three cases showed no enhancement and consequently were not detected by

Table 1 Percentages of benign and malignant lesions in the study population according to histopathology

\begin{tabular}{ll}
\hline & No. $=\mathbf{2 5}$ \\
\hline Benign or malignant & \\
Benign & $11(44.0 \%)$ \\
Malignant & $14(56.0 \%)$ \\
Histopathology & \\
No abnormality & $1(4.0 \%)$ \\
IDC & $13(52.0 \%)$ \\
Fibroadenoma & $5(20.0 \%)$ \\
Lobular mastitis & $2(8.0 \%)$ \\
Lobular carcinoma & $1(4.0 \%)$ \\
Fibroadenosis & $1(4.0 \%)$ \\
Fibrocystic disease & $1(4.0 \%)$ \\
Diabetic mastopathy & $1(4.0 \%)$ \\
\hline
\end{tabular}


Table 2 Demonstration of the added value of CESM in proper detection of lesions

\begin{tabular}{|c|c|c|c|c|c|}
\hline & \multicolumn{2}{|l|}{ CESM detection } & \multirow{3}{*}{$\begin{array}{l}\text { Test } \\
\text { value }\end{array}$} & \multirow{3}{*}{$\begin{array}{l}P \text { - } \\
\text { value }\end{array}$} & \multirow[t]{3}{*}{ Sig. } \\
\hline & Not detected by CESM & Detected by CESM & & & \\
\hline & No. $=3$ & No. $=22$ & & & \\
\hline \multicolumn{6}{|c|}{ Benign or malignant by histopathology } \\
\hline Benign & $3(100.0 \%)$ & $8(36.4 \%)$ & \multirow[t]{2}{*}{4.339} & 0.037 & \multirow{2}{*}{$\begin{array}{l}\text { Significant } \\
\mathrm{S}\end{array}$} \\
\hline Malignant & $0(0.0 \%)$ & $14(63.6 \%)$ & & & \\
\hline
\end{tabular}

CESM, and all were proven benign by histopathology (Table 2).

Regarding the ability of CESM in lesion characterization, twenty-one patients (84\%) were correctly characterized by CESM whether benign or malignant as their results were consistent with the histopathology, with a strong correlation between the diagnosis postulated by CESM and the final histopathological diagnosis $(P$ value $<0.01)$. CESM correctly characterized fourteen patients as having malignant lesions and seven patients as having benign lesions (Table 3). CESM misdiagnosed four cases as BIRADS 4, all of them showed non-mass enhancement, and these cases were proven benign by histopathology; 2 cases of lobular mastitis, 1 case of diabetic mastopathy (Fig. 2), and 1 case of fibroadenoma.

Histopathologically, the results revealed a total of fourteen patients with breast cancer after CESM and tissue core biopsy: thirteen patients with proven IDC (52\%) and one with proven invasive lobular carcinoma (4\%). Among those patients, CESM showed five patients to have multifocal malignancy, three patients had multicentric, and six patients had unifocal malignancy. These diagnoses were confirmed by core needle biopsy. Accordingly, CESM changed the treatment plan from conservative breast surgery to modified radical mastectomy (MRM) +/- neoadjuvant chemotherapy in eight patients (32\% of the total cases included in our study/ $57 \%$ of the patients with histopathologically proven malignancy after CESM and biopsy).

Finally, the diagnostic sensitivity, specificity, positive predictive value (PPV), negative predictive value (NPV), and accuracy of CESM were calculated as $100 \%, 63.6 \%$, $77.8 \%, 100 \%$, and $84 \%$, respectively, in comparison to the diagnostic performance by FFDM; 56\%, 66.6\%, 75\%, $46 \%$, and $60 \%$, respectively (Table 4 ).

\section{Discussion}

Full-field digital mammography is well-established as one of the main diagnostic tools in patients presented with variable breast symptoms especially those presented with clinically palpable breast lumps, yet its sensitivity and specificity are considerably lower in patients with dense breasts. Our study reported the diagnostic sensitivity, specificity, PPV, NPV, and accuracy of FFDM in lesion characterization as $56 \%, 66.6 \%, 75 \%, 46 \%$, and $60 \%$, respectively.

On the other side, CESM is not affected by the breast density as only the enhancing masses will stand out while the rest of the glandular element is represented by background parenchymal enhancement. Our study reported the diagnostic sensitivity, specificity, PPV, NPV, and accuracy of CESM in lesion characterization as $100 \%, 63.6 \%, 77.8 \%, 100 \%$, and $84 \%$, respectively.

Our results were consistent in terms of the high sensitivity of CESM with two studies published in 2020 done by Qin et al. and Zhongflei et al., both studies were concerned with symptomatic patients with dense breasts and reported sensitivity of CESM ranging between $82.4 \%$ and $93.8 \%$ compared to $100 \%$ in our study. Also in another study done by Travesion-Aja et al. in 2019 which is considered one of the largest studies comparing CESM to FFDM in 465 patients with 644 lesions concluded that CESM significantly increased sensitivity compared to FFDM (from 82.5 to 92.3\%) while in our study, CESM increased sensitivity from 56 to $100 \%$ [7-9].

The specificity of CESM calculated from our study $(63.6 \%)$ is lower than the one calculated from other studies done in 2020 by Qin et al. and Zhongflei et al. where it was reported as $96.4 \%$ and $88.1 \%$, respectively; this low specificity in our study is likely attributed to the small

Table 3 Concordance between the diagnoses postulated by CESM and the histopathological diagnoses emphasizing the added value of CESM in accurate characterization of lesions

\begin{tabular}{|c|c|c|c|c|c|}
\hline & \multicolumn{2}{|c|}{ Benign or malignant by histopathology } & \multirow{3}{*}{$\begin{array}{l}\text { Test } \\
\text { value }\end{array}$} & \multirow{3}{*}{$\begin{array}{l}P- \\
\text { value }\end{array}$} & \multirow[t]{3}{*}{ Significance } \\
\hline & Benign & Malignant & & & \\
\hline & No. $=11$ & No. $=14$ & & & \\
\hline \multicolumn{6}{|c|}{ Benign or malignant by CESM } \\
\hline Benign & $7(63.6 \%)$ & $0(0.0 \%)$ & \multirow[t]{2}{*}{12.374} & \multirow[t]{2}{*}{0.000} & \multirow{2}{*}{$\begin{array}{l}\text { Highly significant } \\
\text { HS }\end{array}$} \\
\hline Malignant & $4(36.4 \%)$ & $14(100.0 \%)$ & & & \\
\hline
\end{tabular}



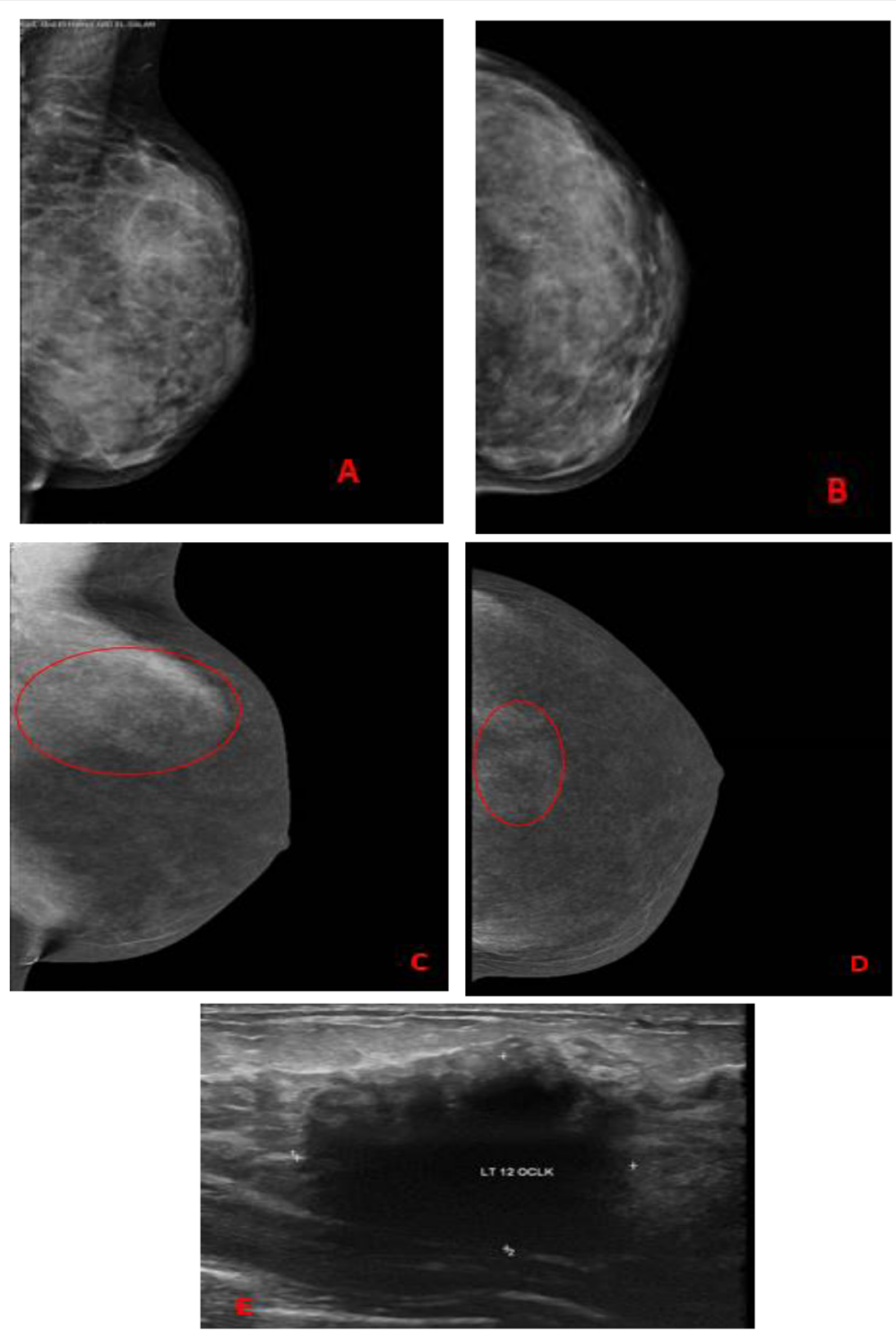

Fig. 2 A 33-year-old female patient presented with left breast lump of 3 months duration. FFDM of the left breast in MLO (a) and CC (b) showing extremely dense breast (ACR D) with no definite masses. CESM of the left breast in MLO (c) and CC (d) showing rather well-circumscribed moderately enhanced mass (circled in c) at the left upper outer quadrant (UOQ) with non-mass enhancement (NME) adjacent to the previously described mass (circled in d). Targeted ultrasound of the left UOQ (i) showing irregular hypoechoic mass lesion. The left breast was categorized after CESM as BIRADS 4A, and biopsy was taken which revealed diabetic mastopathy

sample size (25 patients) compared to a large sample size in these studies which exceeded 100 patients $[7,8]$.

Moreover, our results are consistent in terms of the high sensitivity and low specificity of CESM with the systematic review and meta-analysis study done in 2016 by Tagliafico, et al. which analyzed 604 studies concluding that CESM has high sensitivity (96-100\%) but low specificity (38-77\%), also Luczyńska et al. (2016) concluded low specificity of CESM $(27 \%$ in a study population of 115 patients) [10, 11].

In order to assess the added value of CESM numerically, CESM was assessed from two aspects; the ability to

Table 4 Sensitivity, specificity, positive predictive value (PPV), negative predictive value (NPV), and accuracy of CESM and FFDM

\begin{tabular}{llllllllll}
\hline & TP & TN & FP & FN & Sensitivity & Specificity & PPV & NPV & Accuracy \\
\hline CESM & 14 & 7 & 4 & 0 & $100.0 \%$ & $63.6 \%$ & $77.8 \%$ & $100 \%$ & $84 \%$ \\
FFDM & 9 & 6 & 3 & 7 & $56 \%$ & $66.6 \%$ & $75 \%$ & $46 \%$ & $60 \%$ \\
\hline
\end{tabular}



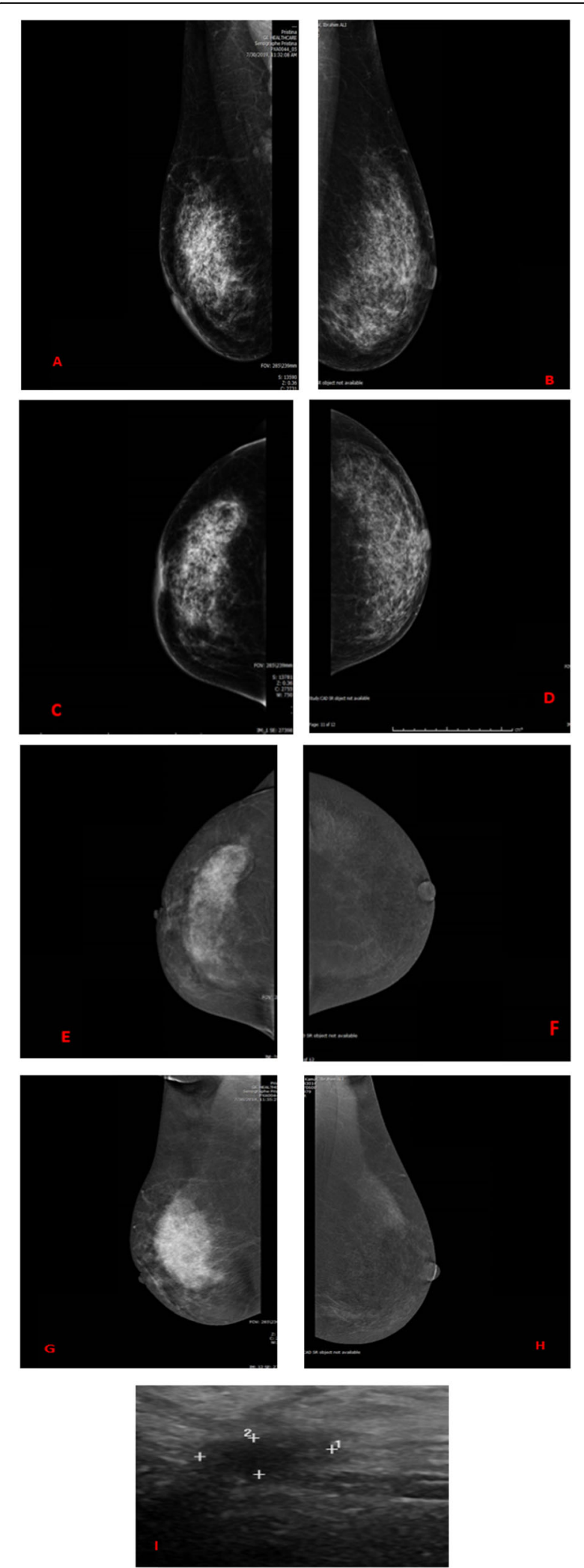

Fig. 3 A 44-year-old female patient presented with left breast lump of 5 months duration, FFDM of both breasts in CC $(\mathbf{a}, \mathbf{b})$ and MLO (c d) showed heterogeneously dense breast (ACR C) with left lower inner quadrant (LIQ) mass (arrowed in $\mathbf{b}$ and $\mathbf{d}$ ) showing partially obscured margin. CESM of both breasts in CC $(\mathbf{e}, \mathbf{f})$ and $\mathrm{MLO}(\mathbf{g}, \mathbf{h})$ revealed multiple intensely heterogeneously enhancing masses (circled in $\mathbf{f}$ and $\mathbf{h}$ ) occupying the whole LIQ; these masses were not obvious on FFDM, the left breast was categorized as BIRADS 5 (multifocal malignancy) while the right breast was categorized as BIRADS 1, and biopsy results from the left LIQ masses revealed invasive ductal carcinoma (IDC)

detect lesions that may have been missed by FFDM due to superimposed heterogeneously dense parenchyma hindering proper visualization of the lesion, and the other aspect was the characterization of lesions as benign or malignant.

In our study, CESM detected the target lesions in twenty-two cases out of twenty-five (88\%). All the lesions detected by CESM were enhancing lesions; eight benign lesions and fourteen malignant lesions. On the other hand, FFDM detected multifocality or multicentricity in malignancy as well as multiple fibroadenomas in only $20 \%$ of the patients, the remaining lesions were identified only by CESM.

Regarding the three cases with clinically palpable lumps that were not detected by CESM as they did not enhance and proved to be benign by histopathology, this point was considered as an advantage for CESM rather than a pitfall; in other words, in these cases, the absence of enhancement was a sign of benignity. The lesions were diagnosed by histopathology and targeted ultrasound as one case of condensed glandular tissue, one case of fibroadenosis, and one case of fibrocystic disease.

CESM correctly characterized 21 cases (84\%) as having benign or malignant lesions in concordance with the final histopathological results. It misdiagnosed only four cases (12\%); those patients were two cases of lobular mastitis, one case of diabetic mastopathy and one case of fibroadenoma. Misdiagnosis was likely attributed to false upstaging non-mass enhancement to BIRADS 4, although the underlying lesions were chronic inflammatory condition or benign fibroepithelial lesion.

This high capability of CESM to detect all lesions and correctly characterize them as benign or malignant was studied by Xing et al. (2019); in a study population of 263 patients with 259 lesions, CESM detected $98.5 \%$ of lesions and correctly characterized or diagnosed $91 \%$ of lesions; the lesions that were not detected by CESM were two lesions of fibroadenoma, one lesion of breast adenosis with ductal dilation, and one lesion of breast adenosis with fibroma formation trend [12].

CESM has a significant role in determining the most appropriate treatment plan specially in cases with histopathologically proven breast cancer where a multifocal 

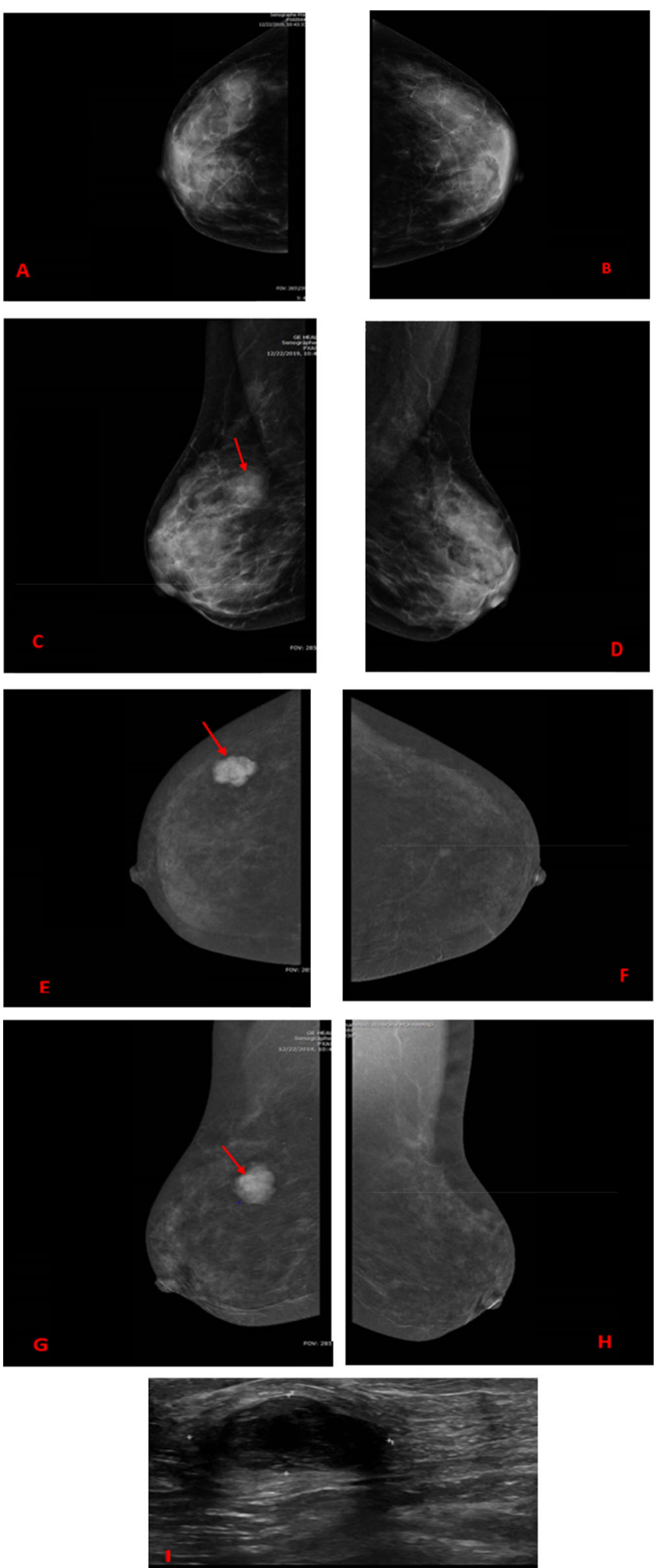

Fig. 4 A 39-year-old female patient presented with right breast lump of 7 months duration being treated as inflammatory changes. FFDM of both breasts in MLO (a and $\mathbf{b}$ ) and CC (c and $\mathbf{d}$ ) showing heterogeneously dense breast (ACR C) with no definite masses yet with right periareolar skin thickening. CESM of both breasts in CC (e and $\mathbf{f}$ ) and MLO ( $\mathbf{g}$ and $\mathbf{h}$ ) showing heterogenous regional non-mass enhancement (NME) involving most of the breast, targeted ultrasound revealed multiple masses (one of them is seen in (i)), ultrasound-guided biopsy was taken and histopathology revealed invasive ductal carcinoma 
or a multicentric malignancy may alter the surgical decision (Figs. 3 and 4); this was observed in $57 \%$ out of fourteen cases diagnosed with breast cancer in agreement with a study done in 2016 by Ali-Mucheru et al. included 100 patients with pathologically proven breast cancer where CESM altered the surgical management to a more extensive surgery in $20 \%$ of these cases [13].

CESM is currently used for the detection of primary breast cancer, the assessment of the extent of disease, a problem-solving tool, and a replacement for MRI where the latter is contraindicated. In terms of patient preferences and tolerance, significantly higher overall preference towards CESM has been demonstrated, due to faster procedure time, greater comfort, and significantly lower rate of anxiety [14].

Concerning the future implications of CESM, preliminary data for the use of CESM in the screening of dense breasts are promising. Mammography is currently the only examination which has been demonstrated to reduce breast cancer mortality, as with all potential screening studies, it is critical to evaluate not only the improvement in sensitivity when using CESM but also if that improvement in sensitivity translates into a decreased number of interval cancers and increases mortality reduction over that of mammography [15].

The key limitation of our study was the small sample size as well as the common limitations of CESM in general which include lack of biopsy capability; if a finding is seen on recombined images only, it can be sampled by finding either a low energy correlates to target with stereotactic/ tomosynthesis-guided core biopsy or an ultrasound correlates to target for ultrasound-guided biopsy. Another limitation relates to the field-of-view of a CESM. Similar to conventional mammography, areas along the chest wall, far medial breast, or in the axilla may not be well-imaged and may be a cause for a false-negative study. Recognizing these limitations is vital, especially if performing CESM in a patient with a palpable abnormality in one of these locations. In these cases, breast MRI may provide a more complete assessment [16].

Our study has deduced that CESM has a great capability to detect and characterize the clinically palpable masses and associated lesions that may be impalpable by clinical examination and missed by FFDM due to obscuration done by heterogeneously dense breast parenchyma, with a significant impact on the choice of the most appropriate treatment plan.

\section{Conclusion}

The added value of contrast-enhanced spectral mammography in dense breasts could be summed up in its value in the detection of lesions that could be obscured on FFDM, more accurate characterization of lesions as suspicious or benign-looking, determining the need for biopsy, and tailoring an adequate treatment plan for the patient.

\section{Abbreviations}

ACR: American College of Radiology; BIRADS: Breast Imaging Reporting and Data system; CC: Craniocaudal; CESM: Contrast-enhanced spectral mammography; FFDM: Full-field digital mammography; IDC: Invasive ductal carcinoma; KVp: Peak kilovoltage; LIQ: Lower inner quadrant; MRI: Magnetic resonance imaging; MRM: Modified radical mastectomy; MLO: Mediolateral oblique; MQSA: Mammography Quality Standards Act; NME: Non-mass enhancement; NPV: Negative predictive value; PPV: Positive predictive value; RI: Recombined images; UOQ: Upper outer quadrant

\section{Acknowledgements}

The authors thank all the study participants for their patience and support.

\section{Authors' contributions}

NNA: Reviewing the literature, data collection and analysis, perform the statistical analysis, write and revise the manuscript, and prepare the figures and tables. SMELG: Reviewing the literature, data analysis, and manuscript editing. RZE: Suggest and develop the research idea, reviewing the literature, and data analysis. EMAH: Reviewing the literature, share in the statistical analysis, data analysis, and manuscript editing. The authors read and approved the final manuscript.

\section{Funding}

This study had no funding from any resource.

\section{Availability of data and materials}

The datasets used and/or analyzed during the current study are available from the corresponding author on reasonable request.

\section{Ethics approval and consent to participate}

The study was a prospective study. This study was approved by the Research Ethics Committee of the Faculty of Medicine at Ain Shams University in Egypt on the 22nd of July 2018; Reference Number of approval: FWA 000017585.

All patients included in this study gave written informed consent to participate in this research. If the patient was unconscious at the time of the study, written informed consent for their participation was given by their legal guardian.

\section{Consent for publication}

All patients included in this research gave written informed consent to publish the data contained within this study. If the patient was unconscious when consent for publication was requested, written informed consent for the publication of this data was given by their legal guardian.

\section{Competing interests}

The authors declare they have no competing interests.

Received: 20 August 2020 Accepted: 29 November 2020

Published online: 05 January 2021

\section{References}

1. Ghoncheh M, Pournamdar Z, Salehiniya H (2016) Incidence and mortality and epidemiology of breast cancer in the world. Asian Pac J Cancer Prev 17(S3):43-46

2. Fallenberg M, Dromain C, Diekmann F et al (2014) Contrast-enhanced spectral mammography versus MRI: initial results in the detection of breast cancer and assessment of tumor size. Eur Radiol 24:256-264

3. Lenihan Megan J, Brem RF (2013) Automated breast ultrasound: a novel approach to screening women with dense breasts. Imaging Med 5(2):139-145

4. Lobbes MBI, Smidt ML, Houwers J (2013) Contrast enhanced mammography: techniques, current results and potential indications. Clin Radiol 68:935-944

5. Mori M, Akashi-Tanaka S, Suzuki S et al (2016) Diagnostic accuracy of contrast-enhanced spectral mammography in comparison to full-field digital mammography in a population of women with dense breasts. Breast Cancer 24:104-110 
6. Kamal RM, Helal MH, Mansour SM et al (2016) Can we apply the MRI BIRADS lexicon morphology descriptors on contrast-enhanced spectral mammography? Br J Radiol 89(1064):20160157

7. Qin Y, Liu Y, Zhang X et al (2020) Contrast-enhanced spectral mammography: a potential exclusion diagnosis modality in dense breast patients. Cancer Med 9:2653-2659

8. Lu Z, Hao C, Pan Y et al (2020) Contrast-enhanced spectral mammography versus ultrasonography: diagnostic performance in symptomatic patients with dense breasts. Korean J Radiol 21(4):442-449

9. Travieso-Aja MM, Maldonado-Saluzzi D, Naranjo-Santana P et al (2019) Diagnostic performance of contrast-enhanced dual-energy spectral mammography (CESM): a retrospective study involving 644 breast lesions. Radiol Med 124:1006-1017

10. Tagliaficoa AS, Bignotti B, Rossic F et al (2016) Diagnostic performance of contrast-enhanced spectral mammography: systematic review and metaanalysis. Breast 28:13-19

11. Łuczyńska E, Niemiec J, Hendrick E et al (2016) Degree of enhancement on Contrast Enhanced Spectral Mammography (CESM) and lesion type on Mammography (MG): comparison based on histological results. Med Sci Monit 22:3886-3893

12. Xing D, Lv Y, Sun B et al (2019) Diagnostic value of contrast-enhanced spectral mammography in comparison to magnetic resonance imaging in breast lesions. J Comput Assist Tomogr 43(2):245-251

13. Ali-Mucheru M, Pockaj B, Patel B et al (2016) Contrast-enhanced digital mammography in the surgical management of breast cancer. Ann Surg Oncol 23:649-655

14. Lotti V, Ravaioli S, Vacondio R et al (2017) Contrast-enhanced spectral mammography in neoadjuvant chemotherapy monitoring: a comparison with breast magnetic resonance imaging. Breast Cancer Res 19(1):106

15. Jochelson MS (2019) Use of contrast-enhanced mammography in breast cancer screening. In: Lobbes M, Jochelson M (eds) Contrast-enhanced mammography. Springer, Cham

16. Phillips J, Fein-Zachary VJ, Slanetz P (2019) Pearls and pitfalls of contrastenhanced mammography. J Breast Imaging 1:64-72

\section{Publisher's Note}

Springer Nature remains neutral with regard to jurisdictional claims in published maps and institutional affiliations.

\section{Submit your manuscript to a SpringerOpen ${ }^{\circ}$ journal and benefit from:}

- Convenient online submission

- Rigorous peer review

- Open access: articles freely available online

- High visibility within the field

- Retaining the copyright to your article

Submit your next manuscript at $\boldsymbol{\nabla}$ springeropen.com 